\title{
Lipomas of the oral cavity: Importance of meticulous clinical evaluation, imaging and histopathological examination for precise treatment planning
}

\author{
Colonel Priya Jeyaraj ${ }^{*}$ and Lt Col Sumeet Sehgal ${ }^{2}$ \\ ${ }^{1}$ Classified Specialist (Oral \& Maxillofacial Surgery), Deputy Commandant, Officer in Charge Trauma \& Rehabilitation, Command Military Dental Centre \\ (Northern Command), Jammu \& Kashmir, India \\ ${ }^{2}$ Classified Specialist (Oral Pathology), Office of Directorate General Dental Services, New Delhi, India
}

\begin{abstract}
Lipomas are benign, slow growing, soft tissue mesenchymal tumors, usually involving the trunk and proximal parts of extremities and occasionally found within the oral cavity in locations such as the buccal mucosa, tongue, floor of mouth, palate, gingiva, lips and retromolar area. Their presentation may vary, as in size, shape, colour, consistency and depth. They are of different histological variants, commonest of them being the Simple Classical lipoma and the Fibrolipoma, and the treatment is complete surgical excision. A case series is presented, showcasing the varieties of lipomas involving different sites within the oral cavity. The importance of histopathological identification of this pathology is important to differentiate it from other look-alike entities affecting similar locations, so as formulate and institute the correct treatment.
\end{abstract}

\section{Introduction}

Lipomas are benign soft tissue neoplasms of mesenchymal origin, composed of adipose tissue. They are asymptomatic, slow growing, smooth- surfaced, lobulated, usually well circumscribed and encapsulated, sessile or pedunculated submucosal masses of variable sizes, although usually less than $30 \mathrm{~mm}$ in diameter [1]. Being the commonest mesenchymal tumors of the trunk and proximal parts of the extremities, $15-20 \%$ cases have been reported to involve the head and neck region as well. However, they are rarely found in the oral cavity comprising just about $1-4 \%$ of benign neoplasms reported in this location, so far [1]. Anatomic sites within the mouth include the buccal mucosa, tongue, floor of mouth, palate, gingiva, lips and Retromolar area [2].

Histologically, Lipomas are classified as Simple lipomas or variants such as Fibrolipoma, Spindle cell lipoma, Intramuscular or infiltrating lipoma, Angiolipoma, Salivary gland lipoma (Sialolipoma), Pleomorphic lipoma, Myxoid and Atypical lipomas [3]. Of these, the Simple lipomas and Fibrolipomas are the commonest. The fibrolipoma is characterized by a significant fibrous component intermixed with the lobules of fat cells. The angiolipoma consists of an admixture of mature fat and numerous small blood vessels. Myxoid lipoma exhibits a mucoid background and may be confused with myxoid liposarcomas. Spindle cell lipomas demonstrate variable amounts of uniform appearing spindle cells together with a lipomatous content. Pleomorphic lipomas are characterized by presence of spindle cells and bizarre hyperchromatic giant cells. Intramuscular lipomas are often more deeply situated and show an infiltrative tendency, extending between skeletal muscle bundles [4].

\section{Case reports}

\section{Case 1}

A 64 year old female patient reported with the complaints of a longstanding swelling below the tongue, which she had first noticed two years ago as a small painless nodule, which had progressively increased in size and was presently causing discomfort and difficulty in speaking, eating, drinking and swallowing. The swelling was not associated with pain, bleeding, or episodes of rupture and discharge. No change in size of the swelling was noted by the patient during meal times.

On examination, the patient was completely edentulous, the teeth having been extracted over the years at various dental clinics for dental caries and chronic destructive periodontitis. At rest, the lesion resembled a light pink colored dome in the right lingual sulcus region, with smooth borders which blended with the adjacent soft tissues, lifting the right half and lateral border of the tongue (Figure 1A). It was roughly ovoid in shape, measuring approximately $5 \mathrm{~cm} \times 5.5 \mathrm{~cm}$, extending from the lower incisor region to the right molar region and crossing the midline of the ventral surface of the tongue to the opposite side of the lingual frenum. The swelling bulged out and became more prominent and accentuated when the patient raised or protruded her

Correspondence to: Colonel Priya Jeyaraj, Classified Specialist (Oral \& Maxillofacial Surgery), Deputy Commandant, Officer in Charge Trauma \& Rehabilitation, Command Military Dental Centre (Northern Command), Jammu \& Kashmir, India, Tel: (91)9596840303, E-mail: jeyarajpriya@yahoo.com

Key words: lipoma, mesenchymal tumors, floor of mouth (FOM)

Received: June 04, 2017; Accepted: June 26, 2017; Published: June 30, 2017 
tongue or rotated her tongue to the left (Figure 1B). There was a mild limitation in tongue movements, especially towards the right, however, taste and somatic sensations were intact. A few superficial blood vessels were visible through the mucous membrane overlying the swelling. On palpation, the swelling was soft, non-tender, compressible and nonfluctuant, and the margins were slippery under the palpating finger. The overlying mucosa was light pink, smooth, without any secondary changes (Figures $1 \mathrm{C}$ and 1D) and was freely mobile showing no fixity to either the swelling underneath or to the adjacent structures. The swelling showed no fixity to the ventral aspect of the tongue, and was not bimanually palpable in the submandibular region indicating that it was limited below by the mylohyoid muscle and hence restricted in location to the sublingual space. Normal saliva could be expressed from the right submandibular salivary gland and there was no sign of hypoesthesia or paresthesia of the area corresponding to the ipsilateral lingual nerve.

The patient's past medical history was not significant. All the routine hematological parameters were within normal limits. An ultrasonography was suggestive of a soft tissue lesion in the floor of the mouth. An infection or inflammatory lesion was ruled out because of the history, lack of symptoms, and the characteristics of the lesion. A ranula was ruled out as the lesion was not the typical translucent blue, and the overlying mucosa was freely movable independently over the mass. A dermoid cyst was ruled out because of the consistency. A provisional diagnosis of a mesodermal tumor such as lipoma was made. Aspiration was negative. Magnetic resonance imaging (MRI) examination revealed well defined lobulated signal intensity area with smooth outline seen along the floor of the mouth, deep, and anterolateral to the Omohyoid muscle suggestive of lipoma of floor of the mouth. FNAC did not yield any material. A computerized tomography scan without contrast (NCCT) and with contrast enhancement (CECT) (Figure 1E), revealed a well demarcated fat containing lesion in the right half of the floor of the mouth, limited medially by the genioglossus, laterally by the Hyoglossus and inferiorly by the mylohyoid and muscles. No calcification or contrast enhancement was noted in the lesion. The lesion was solid, without relation to the mandibular bone. The oro-, naso- and hypo-pharynx appeared normal and remainder of the head and neck scan did not reveal any other pathology or lymph node enlargement.

Surgical excision of the lesion was carried out intra-orally under general anesthesia (Figure 2). An incision was placed through the mucosa directly above the crest of the swelling, which yielded a soft lobulated, encapsulated yellowish mass bulging out (Figures 2A-2C). It was delicately separated from the surrounding connective tissue by gentle blunt dissection. It was then teased out by a wiping motion with a cotton ball stripping off the mucosal cover which cleaved away quite easily, delivering the encapsulated mass (Figure 2D). A distinct plane of separation developed revealing a distinct capsule around thee mass. It was separated quite easily from the contiguous structures like the sublingual salivary gland and Wharton's duct, by the same wiping motion with a cotton ball. The entire mass was excised in toto (Figure 2 D). Macroscopically, it appeared as a yellowish, lobulated, soft, encapsulated mass measuring approximately $5 \mathrm{~cm} \times 2 \mathrm{~cm} \times 1$ $\mathrm{cm}$ (Figure 3A). After ensuring hemostasis, The Wharton's duct was checked for patency before proceeding with a watertight closure of the wound (Figures 2E and 2F).

After delivering the lobulated mass, one portion of its capsule was split carefully (Figures $3 \mathrm{~B}$ and $3 \mathrm{C}$ ) and a small portion of the lesion was excised from the edge and immersed in a test tube containing water to test

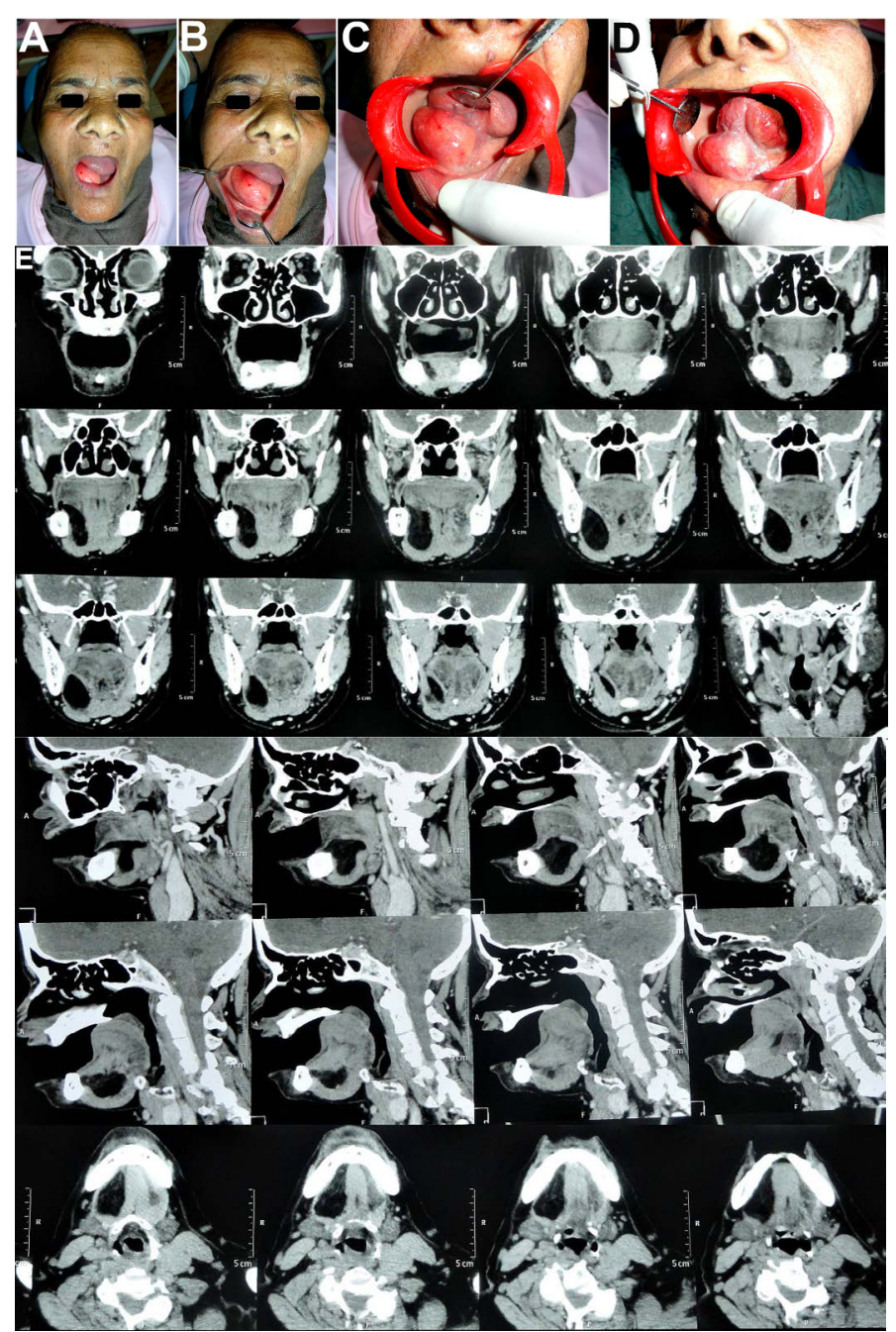

Figure 1. A,B,C,D 64 year old female patient with a longstanding swelling below the tongue, which had progressively increased in size and was presently causing discomfort and difficulty in speaking, eating, drinking and swallowing. E NCCT \& CECT revealed a well demarcated, non-enhancing fat containing lesion in the right half of the floor of the mouth, limited medially by the genioglossus, laterally by the Hyoglossus and inferiorly by the mylohyoid and muscles. Oro-, Naso- and Hypopharynx appeared normal and no other pathology or lymph node enlargement was noted.

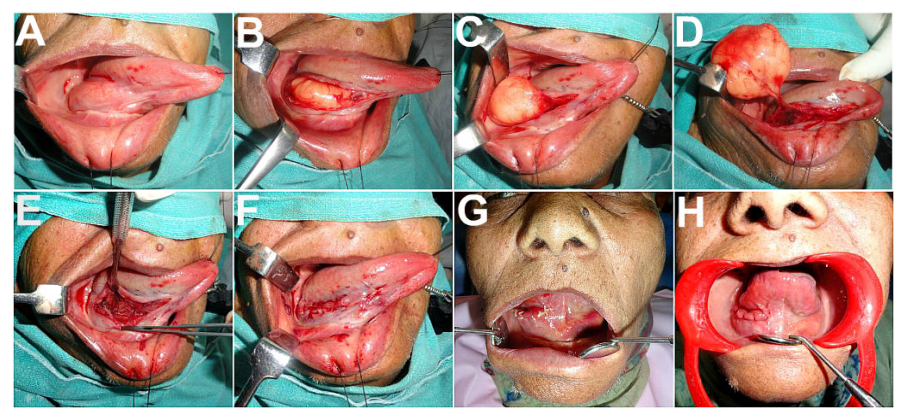

Figure 2. Retraction of the tongue with the aid of a silk suture to aid access and visibility of the lesion. Incision placed through the mucosa directly above the crest of the swelling, which yielded a soft lobulated, encapsulated yellowish mass bulging out. The soft, willowish encapsulated mass was gently teased out, using blunt dissection. It cleaved away readily from the adjacent structures and was excised in toto.

its relative density. It immediately floated to the surface, its buoyancy in water confirming its hyppodensity, reaffirming the presence of fatty tissue, consistent with the provisional diagnosis of a Lipoma. 
The rest of the specimen was sent for histopathological examination, $\mathrm{H} \& \mathrm{E}$ sections of which demonstrated an encapsulated mass consisting of several lobules of mature adipose tissue with sheets of mature fat cells/adipocytes, interspersed with fibrovascular septae (Figures 3D$3 \mathrm{G})$. There was no evidence of nuclear pleomorphism, cellular atypia, hypercellularity, lipoblasts or inflammation, thus confirmative of a benign tumor consistent with the diagnosis of a Simple Lipoma.

Healing was uneventful with no post-operative neurosensory deficits, and no noticeable loss of any of the functions of the tongue or salivary gland or duct (Figure $2 \mathrm{G}$ and $2 \mathrm{H}$ ). The patient was followed up for 18 months with nil recurrence.

\section{Case 2}

A 26 year old male patient reported with the complaint of a painless, longstanding growth on the inside of his left cheek, which had slowly increased in size over 8 months and was now preventing closure of upper and lower lips together and interfering with eating. The swelling was roughly spherical measuring $1 \mathrm{~cm}$ in diameter (Figures 4A-4D), light pink in colour, smooth surfaced, nodular, pedunculated mass with no secondary changes or discharge, soft, non-fluctuant, with margins that were slippery under the palpating finger. The differential diagnosis included lipoma, fibroma, pyogenic granuloma, mucocoel, minor salivary gland tumor. After routine investigations, the patient was taken up for local excision under Local anesthesia. The mass was encapsulated and cleaved away readily from the underlying structures (Figures $4 \mathrm{E}-4 \mathrm{H}$ ). $\mathrm{H}$ \& E sections of which demonstrated an encapsulated mass consisting of lobules of mature adipose tissue with mature fat cells and compressed blood vessels, embedded in a dense collagenous network and fibrovascular septae (Figures 4I-4L). Proliferating fibroblasts were evident in the connective tissue stroma. The overlying epithelium was 8 to 10 layers thick stratified squamous type. The significant fibrous component intermixed with the lobules of fat cells was suggestive of a Fibrolipoma.

The post-operative course was uneventful and no recurrence of the lesion was observed in the entire two years follow-up period.

\section{Case 3}

A 37 year old male patient presented with a large painless swelling below the tongue, which had been present for the past one and a half

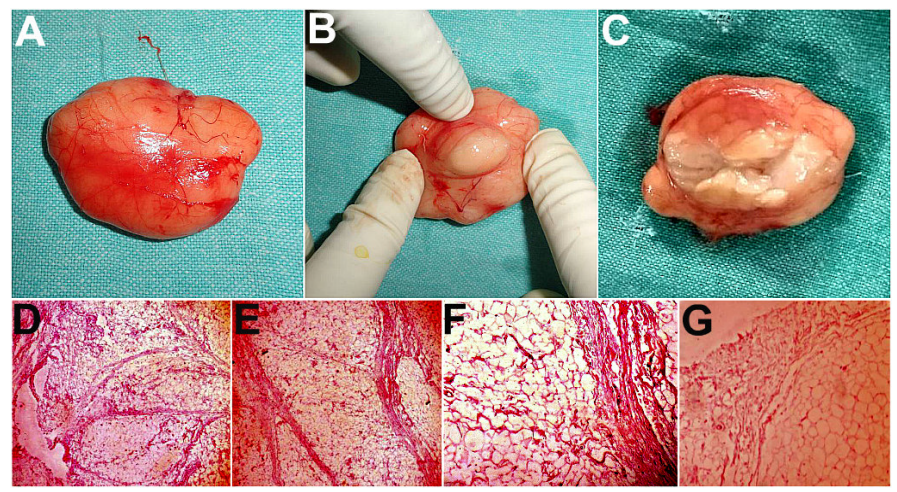

Figure 3. A Macroscopically, the excised specimen appeared as a yellowish, lobulated, soft, encapsulated mass measuring approximately $5 \mathrm{~cm} \times 2 \mathrm{~cm} \times 1 \mathrm{~cm}$. B,C One portion of its capsule was split carefully, and what appeared as fatty expressed out. H\&E Section $10 \mathrm{X}$ and $20 \mathrm{X}$ showed an encapsulated mass consisting of several lobules of mature adipose tissue with sheets of mature round to oval fat cells / adipocytes with peripherally placed nucleus, interspersed with fibrovascular septae, confirming the diagnosis of a lipoma. There was no evidence of nuclear pleomorphism, cellular atypia, hypercellularity, lipoblasts or inflammation.

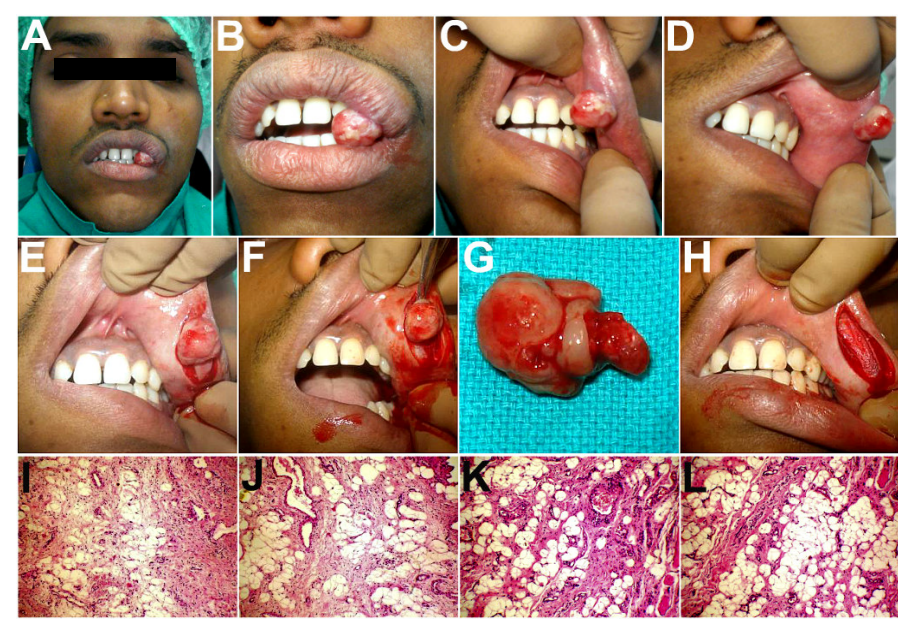

Figure 4. A,B,C,D 26 year old patient with a painless, longstanding roughly spherical growth on the inside of his left cheek. E,F,G,H Surgical excision carried out under Local Anesthesia, with the encapsulated mass cleaving away readily from the underlying structures. I,J,K,L H \& E (x100) Sections showing the mass consisting of lobules of mature adipose tissue with mature fat cells and compressed blood vessels, embedded in a dense collagenous network and fibrovascular septae. A few striated muscle fibres and a few blood vessels containing lymphocytes and mast cells were evident. Proliferating fibroblasts were evident in the connective tissue stroma. The significant fibrous component intermixed with the lobules of fat cells was suggestive of a Fibrolipoma.

years, slowly increasing in size. Since the past 6 months, it had caused some difficulty in speech and swallowing. On examination, there was a single, localized, sessile, spherical swelling in the anterior midline of the floor of the mouth, measuring $8 \times 8 \mathrm{~cm}$ (Figure $5 \mathrm{~A}$ ). The tongue and floor of the mouth were raised, by this solitary, well circumscribed, dome shaped midline swelling extending from the lingual aspect of the mucogingival junction of mandibular anterior teeth up to the mandibular molars bilaterally (Figure 5A). The surface of the swelling was smooth, borders indistinct and the overlying mucosa appeared normal with no secondary changes. The swelling was soft in consistency, non-tender, compressible but non-reducible and with no evidence of inflammation, fluid thrill or impulse on coughing, and was not associated with any discharge. The morphology of the swelling did not vary with tongue movement. Submandibular and sublingual gland orifices appeared normal and bilateral milking of the glands produced normal appearing, mucous saliva. The clinical impression was that of a sublingual dermoid cyst, while the differential diagnosis included a large lipoma, plunging ranula, epidermoid cyst, thyroglossal duct cyst, branchial cleft cyst, cystic hygroma, submental/sublingual space infection or lymph node enlargement, teratoid cyst, sublingual salivary gland infection or tumor.

Mandibular occlusal/cross sectional radiographs did not disclose any calcifications. The buccal and lingual cortical plates were normal with no indication of expansion or decortication. CT scans (axial, coronal and sagittal sections) revealed a large radiolucent space occupying lesion in the sublingual space (Figures 5B-5D). Aspiration of the cyst did not yield anything, suggestive of a solid tumor, possibly a mesenchymal one in view of the soft consistency, such as a lipoma. The patient was taken up for surgical excision of the mass under GA (Figures $5 \mathrm{E}$ and $5 \mathrm{~F}$ ). Adrenaline, 1:100,000 was infiltrated into the floor of the mouth. A suture was placed through the tip of the tongue, which was then retracted upwards (Figure $5 \mathrm{E}$ ). An inverted " $\mathrm{T}$ " shaped incision was made in the midline across the anterior part of the swelling, taking care to avoid any damage to the Wharton's duct and its opening on both sides. Careful submucosal dissection revealed the encapsulated, bulbous, yellowish mass, which was excised in toto by blunt dissection 


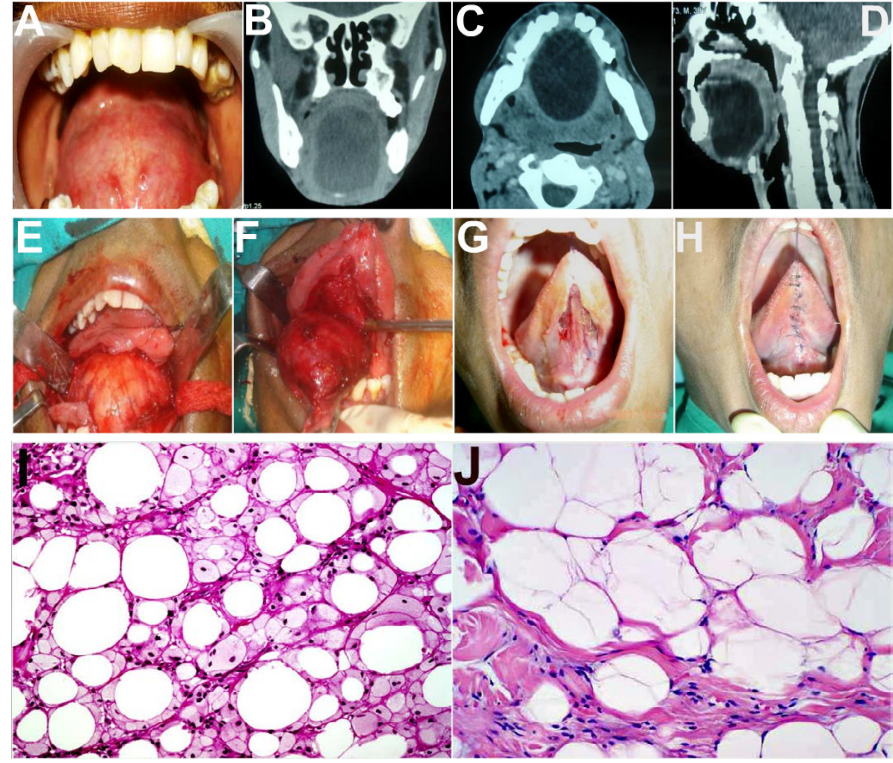

Figure 5. A A $8 \times 8 \mathrm{~cm}$ swelling in the sublingual region of a 35 year old patient raising the tongue upwards. Also seen are the punctate of the openings of the Wharton's ducts. The large swelling caused difficulty in swallowing, eating and talking. B,C,D Axial, Sagittal and Coronal sections of the CT scan revealed a large well circumscribed radiolucency in the sublingual region extending into the submental region, displacing the tongue upwards and causing a narrowing of the upper airway space. E,F An intraoral, inverted T- shaped midline incision from base of the tongue to floor of the mouth, providing excellent exposure of the lesion, followed by blunt pericapsular dissection in an avascular plane. H\&E Section $10 \mathrm{X}$ and 20X showed mature adipose tissue with large adipocytes showing uniformly sized cytoplasmic vacuoles and no atypia. Fibrovascular septae were seen interspersed between the sheets of fat cells.

from the adjacent structures, to which it showed no adhesions, and could be shelled out easily (Figures $5 \mathrm{E}$ and $5 \mathrm{~F}$ ). The openings of the Wharton's ducts were checked for patency followed by closure of the surgical site (Figures 5G and 5H). Post-operative recovery was smooth and uneventful and there was no recurrence of the lesion in the entire two years follow up period.

Histopathological examination of the excised specimen showed mature adipose tissue with large round to oval mature adipocytes, with peripherally places nucleus, uniformly sized cytoplasmic vacuoles and no atypia. Fibrovascular septae were seen interspersed between the sheets of fat cells.

\section{Discussion}

The first description of an oral lipoma was given by Roux in 1848 [5], which he referred to as "yellow epulis". Lipomas are benign soft tissue neoplasms of mature adipose tissue rarely seen in the oral cavity. Though lipomas are the most common tumors affecting the trunk and extremities of the body, their prevalence in the oral cavity varies from just $1-4 \%$ of all tumors seen.

Though the etiology of these tumors is not understood, various theories have been put forward [6]. These include hereditary, hormonal imbalance, fatty degeneration, trauma induced, metaplasia of muscle cells, lipoblastic embryonic cell nest origin, chronic irritation, irradiation, infarction, infection and induction by undifferentiated mesenchymal cells among others. An increased incidence is also seen in patients with high serum cholesterol. However, the reduction in serum cholesterol has not been seen to result in regression of lipomas. Cytogenic abnormalities have also been identified in solitary lipomas. The most common aberration involves translocations between 12q13-
15 and various other chromosomes, most commonly chromosomes 1 , 2,3 , and 21 . The less commonly involved cytogenetic abnormalities in lipomas include interstitial deletions of 13q51,76 and translocations involving chromosome 6p21-23.

Oral lipomas generally occur after the $4^{\text {th }}$ decade of life and show a mild predilection for males. Clinically, they usually present as well demarcated, slowly growing, painless, submucosal swellings which may appear yellowish in colour or similar in colour to the surrounding mucosa depending on the thickness of the overlying epithelium. 2 out of 3 cases in our series presented as swellings in the floor of the mouth and one involved the buccal mucosa on the inner aspect of the corner of the mouth (Table 1). Most oral lipomas are less than $20 \mathrm{~mm}$ in diameter which may be due to the restricted scope of expansion due to the limitation in area available, except in the case of floor of the mouth lesions. In our study, we found that floor of the mouth lipomas were greater than 50 X $50 \mathrm{~mm}$ in dimensions. Great care has to be exercised in excising the lesions from this site owing to rich vascularity and proximity to the Sublingual salivary gland, Wharton's duct and the Lingual nerve.

Due to the benign clinical presentation and minimum discomfort to the patient, many lipomas may remain undiagnosed till they reach a large enough size so as to cause a functional or esthetic problem for the patient. It is probable that the reported incidence of lipomas could be lower than the actual incidence.

The buccal region is the most common site of occurrence of oral lipomas followed by the tongue, lips, floor of the mouth, palate, and gingival [1]. This pattern of predilection also corresponds to the quantity of fat present at the location. We found that out of 3 cases, 2 were present on the floor of the mouth and 1 involved the buccal mucosa. However, due to the limited number of cases, the distribution may not be a true representation of the predilection.

The gold standard for definitive diagnosis of lipomas is the routine Haematoxylin \& Eosin stain [1]. Differentiation from normal adipose tissue is based on the appearance of a circumscribed often encapsulated lesion with absence of vascularity. The adipose cells in a lipoma are usually much larger than in normal adipose tissue and may be upto 200 micron in size. Cartilaginous or osseous metaplasia is rare and may be seen in lipomas of large size and long duration. Secondary changes may occur as a result of trauma or infection which would cause necrosis and liquefaction of fat leading to formation of lipid cysts. Such cases would also show the presence of multinucleated giant cells, indulging in phagocytic activity accompanied by predominantly lymphocytes and a few plasma cells.

On microscopic examination, oral Lipomas have an admixed component of various mesenchymal elements [7]. Depending upon the elements present, the lipoma has been classified into classic/simple lipoma, fibro lipoma, angiolipoma, spindle cell lipoma, pleomorphic lipoma, myxoid lipoma, sialolipoma, and intramuscular lipoma. Most commonly, lipomas exhibit fibrous connective tissue, which is often hyalinised, intermixed with lobules of adipocytes, these lipomas are termed fibrolipomas [4]. When there is a presence of multiple thin walled vascular channels, the lesion is termed angiolipoma. Lesions showing presence of uniform spindle shaped cells among normal adipocytes are termed spindle cell lipomas. When spindle cells show dysplastic features or are mixed with pleomorphic giant cells the lesion is termed pleomorphic lipomas. Lesions with a myxoid background stroma are termed myxoid lipomas. When the lipoma infiltrates surrounding striated muscle tissue, it is called an intramuscular lipoma. 
Table 1. Clinical findings.

\begin{tabular}{|c|c|c|c|c|c|c|}
\hline S. No & $\begin{array}{c}\text { Age } \\
\text { (in yrs) }\end{array}$ & $\begin{array}{l}\text { Sex } \\
(\mathbf{M} / \mathbf{F})\end{array}$ & Site & $\begin{array}{c}\text { Size } \\
\text { (in } \mathbf{m m s} \text { ) }\end{array}$ & $\begin{array}{l}\text { Duration } \\
\text { (In months) }\end{array}$ & Chief complaints \\
\hline 1. & 64 & $\mathrm{~F}$ & Floor of mouth & $5 \times 5.5$ & 24 & $\begin{array}{l}\text { Discomfort and difficulty experienced in speaking, eating, drinking } \\
\text { and swallowing. }\end{array}$ \\
\hline 2. & 26 & M & $\begin{array}{l}\text { Buccal mucosa just inside the } \\
\text { left angle of the mouth }\end{array}$ & $2 \times 2$ & 08 & $\begin{array}{l}\text { Preventing closure of upper and lower lips together, frequently } \\
\text { traumatized by the anterior teeth and interfering with eating }\end{array}$ \\
\hline 3. & 37 & M & Floor of mouth & $6 \times 6$ & 30 & $\begin{array}{l}\text { Tongue raised upwards towards the palate, discomfort and difficulty } \\
\text { experienced in speaking, eating, drinking and swallowing. }\end{array}$ \\
\hline
\end{tabular}

Diagnosis of the Lipoma may present many interesting challenges [8]. There are a variety of lesions and conditions to be considered while diagnosing a case of intra oral lipoma, owing to similarities in their presentation. Differential diagnoses of lesions responsible for enlargements in the oral cavity, especially in the region of the floor of the mouth include:

a) Infectious/Inflammatory lesions: Sublingual space infections and abscesses, Submandibular or submental Lymphadenitis (TB scrofula, Actinomycosis or Cat Scratch disease), Sialadenitis.

b) Traumatic/Obstructive Lesions: Mucus retention/extravasation phenomenon such as Ranula/Mucocoel

c) Developmental lesions: Dermoid cyst, Epidermoid (Epidermal inclusion) cyst, Lymphoepithelial cyst, Thyroglossal duct cyst, Branchial cleft cyst, Heterotopic gastrointestinal cysts, Cystic hygroma (lymphangioma), Ectopic Gastrointestinal cyst.

d) Congenital lesions: Hemangiomas and Vascular malformations, Lymphangiomas.

e) Neoplastic Lesions: Lipoma, Granular Cell Tumor, Rhabdomyoma, Neuroma, Neurofibroma, Salivary gland tumors such as mixed tumors, mucoepidermoid carcinoma and adenocarcinoma, Benign mesenchymal tumors, Lymphoma (Hodgkin's/Non-Hodgkin's).

Developmental lesions are generally located in the midline and the age of the patient at presentation, is usually much younger. More importantly the onset of symptoms and duration of existence help in ease of differentiation from a lipoma. Commonest among the developmental lesions of the oral cavity are the Epidermoid or epidermal inclusion cysts, frequently seen in the floor of the mouth, caused by the impalement of the ectoderm at the time of closure of embryonic fissures. They usually contain sebaceous glands and sebaceous material, however, may also contain hair or hair follicles. While they are present at birth they usually do not become evident till later years, with their growth becoming accelerated during puberty. Congenital lesions such as, Vascular Malformations, Hemangiomas and Lymphangiomas present at a much earlier age and overlying mucosa is discolored. Infectious and inflammatory conditions can be ruled out in the absence of constitutional symptoms such as fever, malaise and tenderness over the lesion [9]. Further, in the case of lipomas, the overlying mucosa appears normal, with no secondary changes or discharge. Obstruction of salivary ducts or extravasation from them leads to a fluid filled swelling, which may be either a mucocoel or ranula, both of which yield a positive aspirate. A non-tender, slowly growing swelling with lack of nodal involvement and normal overlying mucosa can exclude malignant lesions.

The diagnosis of oral lipomas is usually clinical. Further, MRI and CT imaging assist in diagnosing and evaluating them. MR imaging depicts fatty lesions with high signal intensity on T1- weighted images and signal loss on fat-saturated images. CT shows well-defined lesions with the low attenuation of fat. Histopathology remains the Gold standard in the definitive diagnosis of the lipoma [10].
The treatment for oral lipomas, irrespective of the location, dimension, histological presentation or classification remains complete surgical excision. Recurrence of oral lipomas is rare. The very few cases of recurrence reported, could be due to the incomplete excision of deep seated tumors, as in the case of large lipomas of the floor of the mouth or due to their proximity to vital structures in the area. In our series, after a period of 2 years there was no incidence of recurrence in any of the cases.

\section{Conclusion}

The importance of meticulous clinical evaluation and histopathological identification of the Lipoma is important to differentiate it from other look-alike entities affecting similar locations, so as institute the correct treatment. In particular, lesions and enlargements in the floor of the mouth often pose a significant diagnostic challenge, as a variety of conditions ranging from the innocuous ranula to the sinister carcinoma have similar clinical presentations and findings. The importance of investigations such as CT scans, MRI, and Histopathological confirmation cannot be stressed enough while dealing with these entities to prevent possible misdiagnosis and wrong surgical planning, such excision of the sublingual or submandibular salivary gland. Complete surgical excision of the lipoma is the definitive treatment modality to be employed and if performed with care, provides an excellent outcome and is not followed by recurrence.

\section{Compliance with ethical standards \\ Disclosure of potential conflicts of interest}

The author of this article has not received any research grant, remuneration, or speaker honorarium from any company or committee whatsoever, and neither owns any stock in any company. The author declares that she does not have any conflict of interest.

\section{Research involving human participants and/or animals}

All procedures performed on the patients (human participants) involved were in accordance with the ethical standards of the institution and/or national research committee, as well as with the 1964 Helsinki declaration and its later amendments and comparable ethical standards.

\section{Ethical approval}

This article does not contain any new studies with human participants or animals performed by the author. Informed consent

Informed consent was obtained from all the individual participants in this study.

\section{Funding}

This study was not funded by any organization/society. 


\section{References}

1. Fregnani ER, Pires FR, Falzoni R, Lopes MA, Vargas PA (2003) Lipomas of the ora cavity: clinical findings, histological classification and proliferative activity of 46 cases. Int J Oral MaxillofacSurg32: 49-53.[Crossref]

2. Manor E, Sion-Vardy N, Joshua BZ, Bodner L (2011) Oral lipoma: Analysis of 58 new cases and review of the literature. Ann DiagnPathol15:257-261.

3. Neville BW, Damm DD, Allen CM, Bouquot JE (2004) Oral \&maxillofacial pathology. 2nd ed, Philadelphia: Elsevier publication.

4. Furlong MA, Fanburg-Smith JC, Childers EL (2004) Lipoma of the oral and maxillofacial region: Site and subclassification of 125 cases. Oral Surg Oral Med Oral Pathol Oral RadiolEndod98: 441-450.[Crossref]
5. Agarwal P, Chaudhary M (2014) A rare case of intraoral lipoma in a 33 months old child and a review. Dentistry4: 215-216

6. Ranginwala A, Kale H, Modi T, Dave K (2010) Intra-oral lipoma. J IntClin Dent Res Organ 2: $157-160$

7. Kumar P, Naraniya A (2012) Intraoral fibrolipoma: A rare histological variant. Indian J Oral Sci3: $39-41$

8. Tan MS, Singh B (2004) Difficulties in diagnosing lesions in the floor of the mouth reportof two rare cases. Ann Acad Med Singapore 33: 72S-76S.

9. Raj AA, Shetty PM, Yadav SK (2014) Lipoma of the floor of the mouth: report of an unusually large lesion. J Maxillofac Oral Surg13: 328-331.[Crossref]

10. Patil A, Patil K, Tupsakare S, Ali FM (2014) Intraoral lipoma at the floor of the mouth A case report. International Journal of Pharmacology and Therapeutics4: 2249-2250.

Copyright: $\odot 2017$ Jeyaraj CP. This is an open-access article distributed under the terms of the Creative Commons Attribution License, which permits unrestricted use, distribution, and reproduction in any medium, provided the original author and source are credited. 